\title{
Capsule Commentary on Aikens et al., Integrating Support Persons into Diabetes Telemonitoring to Improve Self-Management and Medication Adherence
}

\author{
Amir Mohammad, MD, MPH \\ VA Connecticut Healthcare System, Yale University School of Medicine, West Haven, CT, USA.
}

J Gen Intern Med 30(3):346

DOI: $10.1007 / \mathrm{s} 11606-014-3122-4$

(c) Society of General Internal Medicine 2015

$\mathrm{T}$ his study conducted by Aikens et al. ${ }^{1}$ explored the potential benefits for medication adherence among diabetics after integrating a support person (relative or friend) into a VA-based telemonitoring program. These patients participated in 3-6 months of weekly telemonitoring via Interactive Voice Response Calls. Patients had the options of designating a care partner. After each interactive voice response (IVR) call that the patient completed, these care partners received an automatically generated email summarizing patient's general health and self-management status. Clinicians were also notified about their patient's nonadherence response via an automatically generated fax. The authors computed the physical composite summary, mental composite summary, and measured long-term medication adherence with the validated Morisky Medication Adherence Scale. They concluded that diabetics who were both noncompliant and psychologically distressed were readily engaged with automated telehealth. Another interesting observation was the selection and involvement of the care partner selected by the patient who received emails and text messages after IVR calls.

Despite recruiting from 16 geographically different VA clinics, the authors were only able to recruit 98 non-adherent diabetic patients, who opted to involve a care partner. These participants were not selected randomly and their responses about adherence were purely subjective. Therefore, the results are less convincing due to small sample size and limited duration of the study.
Previous studies have examined the impact of telemonitoring on discrete clinical outcomes such as a decrease in HbAlc. ${ }^{2}$ However, an observational study conducted by Chumbler et al. didn't find any significant clinical impact of using telehealth monitoring of veterans' diabetes when comparing patients monitored on a daily basis versus patients monitored weekly. ${ }^{3}$

Despite these limitations, these preliminary findings should encourage clinicians and specialists to involve a support person at an early stage when evaluating noncompliant diabetics. It is worth mentioning that the VA has been a leader in providing services for veterans with chronic illnesses including diabetes. Future randomized controlled trials should include telemonitoring of diabetic patients with and without a support person and focus on measuring the diagnostic values related to diabetes complications and healthcare utilization.

Conflict of Interest: The author has no conflict with any of the material in this manuscript.

Corresponding Author: Amir Mohammad, MD, MPH; VA Connecticut Healthcare System, Yale University School of Medicine, West Haven, CT 06516, USA (e-mail: amir.mohammad@yale.edu).

\section{REFERENCES}

1. Aikens JE, Trivedi R, Arron DC, Piette JD. Integrating Support Persons into Diabetes Telemonitoring to Improve Self-Management and Medication Adherence. J Gen Intern Med. 2014. doi:10.1007/s11606-014-3101-9.

2. Jaana M, Paré G. Home telemonitoring of patients with diabetes: a systematic assessment of observed effects. J Eval Clin Pract. 2007;13(2):242-53.

3. Chumbler NR, Neugaard B, Kobb R, Ryan P, Gin H, Joo Y. An observational study of veterans with diabetes receiving weekly or daily home telehealth monitoring. Journal of Telemedicine and Telecare. 2005; 11(3): 150-156. 